Service social

\title{
Le bruissement du temps, le dynamisme du vieillissement, Marcelle Brisson, Montréal, Triptyque, 1992, 150 pages.
}

\section{Francine Pinard}

Volume 44, numéro 1, 1995

Valeurs, pratiques, action sociale

URI : https://id.erudit.org/iderudit/706688ar

DOI : https://doi.org/10.7202/706688ar

Aller au sommaire du numéro

Éditeur(s)

École de service social de l'Université Laval

ISSN

1708-1734 (numérique)

Découvrir la revue

Citer ce compte rendu

Pinard, F. (1995). Compte rendu de [Le bruissement du temps, le dynamisme du vieillissement, Marcelle Brisson, Montréal, Triptyque, 1992, 150 pages.] Service social, 44(1), 160-162. https://doi.org/10.7202/706688ar d'utilisation que vous pouvez consulter en ligne.

https://apropos.erudit.org/fr/usagers/politique-dutilisation/ 
Ce qui caractérise cet ouvrage, c'est le fait de présenter aux lecteurs une vision large et diversifiée du phénomène, englobant les dimensions scientifique et pratique; les aspects biologiques, psychologiques et sociaux; les perspectives historique, philosophique et culturelle; les intérêts politiques et économiques; les enjeux idéologiques et éthiques.

Ces volumes s'adressent non seulement aux chercheurs, mais aussi à tous les praticiens sociaux débutants et d'expérience.

René AUCLAIR

Professeur

École de service social, Université Laval

\section{LE BRUISSEMENT DU TEMPS, LE DYNAMISME DU VIEILLISSEMENT}

Marcelle Brisson

Montréal, Triptyque, 1992, 150 pages.

Cet ouvrage est un essai sur le vieillissement, une réflexion de l'auteure au mitan de sa vie, en partant du concept de l'âge. Il s'adresse à un public lettré et sera particulièrement apprécié des chercheurs en gérontologie sociale désireux de trouver une analyse théorique et développementale du vieillissement. Selon l'auteure, le vieillissement en appelle au dynamisme bio-psycho-social de l'être humain et à ses capacités de s'adapter aux différentes étapes du cycle de vie. La vieillesse catégorise les personnes et inscrit l'irréversibilité du passage du temps humain.

Cet ouvrage se partage en deux sections bien distinctes. La première partie, "Dynamisme et vieillissement ", est une analyse théorique de type existentialiste et fait appel à la construction sociale du vieillissement. La deuxième partie, "À chacun d'inventer son vieillissement ", fait état de l'expérience de la retraite de l'auteure et de sa réflexion sur sa propre retraite. Dans l'ensemble la discussion est surtout réflexive, et c'est d'ailleurs la force et l'intérêt de ce livre.

La proposition initiale de l'auteure est de situer le vieillissement dans la dynamique du vivant. Le premier élément de réflexion est la prise de conscience de son vieillissement. La plupart des personnes ne se voient pas vieillir, car le discours sociétal identifie le vieillissement à la vieillesse. Un des écrans est la construction sociale du vieillissement. La société organise notre vie à travers ses institutions (famille, école, travail, etc.). L'âge chronologique sert donc de critère pour l'évaluation des événe- 
ments de la vie et accompagne également les stades traditionnels d'évolution tels la jeunesse, l'âge adulte et la vieillesse. Une autre difficulté est la confusion entre la vieillesse et la maladie, car le vieillissement est souvent associé à la maladie et non pas à un processus évolutif normal. On ne peut parler du vieillissement sans parler de la mort. Par ailleurs, les images positives du vieillissement sont associées à l'augmentation en nombre des gens âgés et à l'allongement de la durée de vie qui favorise l'émergence de nouveaux constats sociaux (tels que la formation scolaire continue, la deuxième carrière, le travail à temps partiel) ainsi qu'à une certaine liberté identifiée aux loisirs. Les associations de nouveaux retraités créent leurs propres organisations sociales et se mobilisent pour influencer les hommes politiques dans le but de défendre leurs droits et leurs libertés individuelles et fondamentales.

Le second élément de réflexion insiste sur l'importance de réaliser qu'on est vivant. Réaliser son vieillissement, c'est favoriser l'action de son dynamisme bio-psycho-social, c'est-à-dire identifier, discerner et reconnaître les pertes subies, se permettre de ne pas réaliser tous ses projets et apprendre à faire des deuils. Pour ce faire, il faut repenser sa vie en fonction de son expérience personnelle, de ses connaissances et de son cadran biologique. C'est à partir de cette étape que l'auteure considère que le dynamisme humain se réalise dans la confiance du vivant de la vie. L'humain intervient et contrôle son milieu et le construit en fonction de ses besoins. La conscience de soi permet de saisir son vieillissement et d'agir sur lui.

Le troisième élément de réflexion invite le lecteur à prendre conscience des différents éléments susceptibles de ralentir le dynamisme humain. Selon l'auteure, la retraite invite à une redéfinition de la notion du temps en lien avec la liberté d'agir. Le rapport au temps devient une réalité paradoxale, c'est-à-dire qu'il s'inscrit dans le sens de la liberté et du plaisir, mais il renvoie aussi à la notion du passé qui s'allonge et du présent qui se rapproche. Cette période de remise en question force une réflexion sur les valeurs personnelles, sur le rôle de l'humain dans une perspective sociale et sociétale, sur la mobilité des structures sociales actuelles ainsi que sur les normes et les valeurs d'une société en mouvance. Dans cet essai, l'auteure croit fermement au dynamisme humain et à sa capacité d'ajustement au passage du temps sur lui.

Ce livre est facile à lire, mais les références à Montaigne et Platon ne sont pas à la portée de tous les lecteurs; par ailleurs, il constitue une initiation aux écrivains et philosophes classiques. La partie théorique fait référence à une vision éclectique du vieillissement, ce qui permet au lecteur de s'initier à une connaissance générale du phénomène. Les éléments de discussion de cet essai font appel à une vision élitiste du vieillissement et obligent à s'interroger sur le «comment » notre clientèle 
vieillit. Cette réflexion suggère une question que les travailleurs sociaux devraient se poser: que pouvons-nous faire pour mieux rejoindre notre clientèle dans ses interrogations face aux angoisses suscitées par le vieillissement?

Francine PINARD

Travailleuse sociale

Hôpital Bellechasse, Montréal Étudiante à la maîtrise en travail social Université Laval, Québec

\section{COMMUNIQUUÉ}

ÉCOLE DE SERVICE SOCIAL FACULTÉ DES SCIENCES SOCIALES

Le Fonds Simone-Paré a été créé à I'Université Laval en 1990 pour promouvoir le développement du service social des groupes.

\section{HOHOH UNIVERSITÉ 通田 LAVAL}

\section{LE SAVOIR DU MONDE PASSE PAR ICI}

\section{Le FONDS SIMONE-PARÉ}

est heureux d'annoncer

qu'il a remis son prix

d'excellence annuel 1995 à madame Isabelle Côté, travailleuse sociale au CLSC Sainte-Foy/Sillery, pour ses interventions auprès de parents divorcés et de leurs enfants. 